EFOMP Policy Statement

\title{
The European Federation of Organisations for Medical Physics Policy Statement No. 10.1: Recommended Guidelines on National Schemes for Continuing Professional Development of Medical Physicists ${ }^{1}$
}

\author{
Stelios Christofides ${ }^{a, *}$, Jorge Isidoro ${ }^{b}$, Csilla Pesznyak ${ }^{c}$, Florian Cremers ${ }^{d}$, Rita Figueira ${ }^{e}$, \\ Christiaan van Swol ${ }^{\mathrm{f}}$, Stephen Evans ${ }^{\mathrm{g}}$, Alberto Torresin ${ }^{\mathrm{h}}$ \\ a Biomedical Research Foundation, P.O. Box 24039, 1700 Nicosia, Cyprus \\ ${ }^{\mathrm{b}}$ Coimbra Hospital and Universitary Centre, 3000-075 Coimbra, Portugal \\ c Department of Technology and Economics, Budapest University, Muegyetem rkp. 3, 1111 Budapest, Hungary \\ d UKSH Lübeck, Ratzeburger Allee 160, 23538 Lübeck, Germany \\ e Centro Hospitalar de São João, Alameda Prof. Hernani Monteiro, 4200-319 Porto, Portugal \\ ${ }^{f}$ St. Antonius Hospital, PO Box 2500, NL-3430 EM Nieuwegein, Netherlands \\ g EFOMP, Fairmount House, 230 Tadcaster Road, York YO24 1ES, UK \\ hedical Physics Dep., Niguarda Ca' Granda Hospital, Milano, Italy
}

\section{A R T I C L E I N F O}

Article history:

Available online 2 February 2016

\begin{abstract}
A B S T R A C T
Continuing Professional Development (CPD) is vital to the medical physics profession if it is to embrace the pace of change occurring in medical practice. As CPD is the planned acquisition of knowledge, experience and skills required for professional practice throughout one's working life it promotes excellence and protects the profession and public against incompetence. Furthermore, CPD is a recommended prerequisite of registration schemes (Caruana et al. 2014 [1]; [2]) and is implied in the Council Directive 2013/59/EURATOM (EU BSS) [3] and the International Basic Safety Standards (BSS) [4]. It is to be noted that currently not all national registration schemes require CPD to maintain the registration status necessary to practise medical physics. Such schemes should consider adopting CPD as a prerequisite for renewing registration after a set period of time.

This EFOMP Policy Statement, which is an amalgamation and an update of the EFOMP Policy Statements No. 8 and No. 10, presents guidelines for the establishment of national schemes for CPD and activities that should be considered for CPD.
\end{abstract}

(C) 2016 Published by Elsevier Ltd on behalf of Associazione Italiana di Fisica Medica.

\section{Introduction}

In March 1997 EFOMP published Policy Statement No. 8, “Continuing Professional Development for the Medical Physicist”, and in October 2000 Policy Statement No. 10, "Recommended Guidelines on National Schemes for Continuing Professional Development of Medical Physicists", making recommendations on CPD for medical physicists, the setting up of CPD schemes and encouraging their implementation by NMOs and the European Societies involved in medical physics activities. The recent developments in medical

${ }^{1}$ Disclaimer Note: EFOMP Policy statement No.10.1 has been approved by EFOMP Council through postal ballot on the 25th of December 2015. Hence this paper has not been subjected to standard peer review, being an official policy statement of the European Federation of Organisations for Medical Physics.

* Corresponding author. Tel.: +357 22322 278; fax: +357 22325415.

E-mail address: c4stelios@gmail.com (S. Christofides). physics $[1,4]$ and the new publication of the EU BSS have necessitated the revision of these two Policy Statements.

The requirement for CPD in the field of medical physics is implied by the EU BSS [3] and it is further elaborated in the European Commission's Radiation Protection Report 174 (RP 174) [5]. A certain level of education and training is recommended to commence clinical practice and to register as a medical physicist. Furthermore CPD builds on this foundation and ensures increasing competence and expertise post registration, so that the medical physicist may be recognised as a Medical Physics Expert (MPE) by the relevant national competent authorities as required by the EU BSS [3]. Furthermore, CPD is required if MPEs are to maintain their recognition.

Whilst the EU BSS is binding only on European Union Member States (EU MS), they do affect every European country. Furthermore, whilst they deal primarily with medical radiation physics, they effectively set the standards for the whole medical physics profession. In the majority of European countries, medical physics services extend beyond the traditional sphere of medical radiation physics, 
encompassing scientific, technical and management support for medical technology throughout the hospital.

The individual medical physicist's CPD should be in line with his/ her professional activities and registration level and his/her ambitions to achieve a higher professional level [3].

Appendix A discusses the necessity for CPD in medical physics and also gives an explanation of what constitutes CPD.

According to the EU BSS, member states are required to ensure that medical physicists have access to continuing education and training after qualification in addition to their basic theoretical and practical training. This policy statement aims to support the EFOMP National Member Organisations (NMO) and the European Societies involved in the organisation of medical physics workshops, conferences and courses to foster the growth of formal, standardised CPD schemes in a harmonised way.

\section{Aims and objectives}

The most important aim of EFOMP is to achieve harmonisation in the qualification of medical physicists in Europe, through the establishment of education, training and CPD schemes according to a set of basic recommendations.

Regarding lifelong learning, the objectives of these guidelines are:

- To promote CPD in the medical physics community

- To provide a framework, which allows the NMOs to create their own CPD schemes

- To guide the NMOs in establishing a credit system for CPD

- To ensure harmonisation across Europe in the assessment of CPD related activities.

All medical physicists should be involved in CPD after their registration as medical physicists or their recognition as MPEs. Whilst EFOMP recognises that it has no statutory authority in this area, it fully supports CPD undertaken on a voluntary basis (or regulated by national rules) as a practical contribution to enhancing patient care and therefore invites its NMOs to implement the requirements of this Policy Statement in the situations where statutory obligations do not exist.

CPD programmes have been and are being developed on the national level in the medical physics community. These may be credit based or outcome based (e.g. portfolio). EFOMP NMOs have different work practices and training systems. EFOMP Guidelines presented here are for a credit based CPD scheme. They are intentionally flexible, thus allowing the NMOs to set up their own detailed CPD schemes within this framework and under their present local and national circumstances and constraints. If requested, EFOMP can provide professional advice to NMOs in setting up their national CPD schemes.

The concept of CPD is related to the knowledge, skill and competence acquired during lifelong learning rather than to the amount of time used to acquire them (see Appendix A). The outcome of CPD should lead to an improvement in professional practice. As qualitative outcome based systems are developed, these guidelines may be revised.

\section{Recommended guidelines for national schemes for CPD}

All NMOs should develop their own detailed CPD scheme according to the following general requirements. Each CPD scheme should be based on a quantitative assessment of the individual's CPD activities. Both the CPD scheme and the credit point system recommended here are intentionally very general and flexible, and NMOs are challenged to design their own CPD schemes and credit point systems to meet their local requirements.
The national CPD schemes should be part of the national registration schemes for medical physicists as recommended by the EFOMP Policy Statement No. 6.1, "Recommended Guidelines on National Registration Schemes for Medical Physicists” [2].

\section{General requirements of national CPD schemes}

Formal CPD schemes require some methods of quantifying CPD. This may be based on numeric values ("CPD Points") for activities which contribute towards, or result from, CPD. The scheme can then measure individual performance against a target (e.g. required number of "CPD points" to be achieved over a set period).

CPD schemes can be based only on the evaluation of evidence derived from the "personal CPD cycle" (see Appendix A), without the need to collect CPD points. An example of such a scheme is that of the United Kingdom, run by the Health and Care Professions Council - HCPC (for more details on this type of CPD schemes please visit the HCPC website at http://www.hpc-uk.org/) and it is based on the following broad categories of activities:
- Work based learning
- Professional activity
- Formal/educational
- Self directed learning
- Other

CPD schemes can also be a combination of the above two types of CPD schemes. Each national CPD scheme must be designed to meet the requirements of the particular country.

Registration schemes require evidence of continued activity in medical physics for registration renewal and CPD schemes provide a mechanism for monitoring such activity. Therefore these requirements for continued endeavour throughout professional life will enhance the status of the profession and its value to the patient and the employer.

As mentioned previously, the most important aspect of CPD is the outcome of the CPD activity rather than the length of time involved in its participation (see Appendix A).

For demonstration purposes, some ideas are presented below based on a two category activities quantitative CPD scheme, usually preferred as the starting point of newly established CPD schemes.

Such schemes eventually evolve to the "personal CPD cycle" type as such schemes represent the essence of CPD.

\section{Description of the quantitative CPD schemes}

The credit point ( $\mathrm{cp}$ ) is the unit of CPD; $1 \mathrm{cp}$ typically corresponds to one full hour of educational activity. To maintain professional competence, 40-60 cp of formally agreed and recorded CPD should be undertaken per annum. The CPD scheme should be based on a 5-year cycle with a total of 200-300 cp. Because circumstances vary from time to time and with them the opportunity to earn CPD points, NMOs may feel that it is appropriate to maintain a five year rolling average of $40-60 \mathrm{cp}$ per annum. The minimum time of professional activity within the 5 year cycle, required for the renewal of registration, should be specified by the NMO. CPD activities should be classified into two categories:

Category 1 (see 3.2.1) activities are attendance at pre-assessed training events, i.e. lectures, scientific meetings, workshops, refresher and training courses. The assessment of the training event should be related to its contents and relevance and should be determined by the NMO.

Category 2 (see 3.2.2) activities are different types of planned and agreed self-directed learning tasks. 
Notification of current CPD status

Registration schemes should develop a model for recording the credit points for the individual registered medical physicist.

All medical physicists and MPEs enrolled in CPD should report their CPD credit point records to their registration schemes for validation and record keeping.

\section{Requirements on the credit point system}

- 1 cp typically corresponds to 45-60 minutes of educational activity in Category 1. Since category 2 activities are diverse in character, there is no similar simple equivalence between credit points and hours spent.

- The 200-300 cp per five (5) year cycle should be achieved as a mixture of Category 1 and 2 activities as prescribed below, with a minimum cp required for each category.

- Courses organised by other NMOs, specialist groups, or at an international level, where the course content is relevant to medical physics practice and where there is prior CPD approval by the hosting organisation, should give credit points recognised on that same basis.

- Credit points in excess of the required 200-300 cp should not be carried forward into the following cycle of five years, unless a system of a rolling five year average has been adopted.

\section{Category 1 credit points}

- NMOs should require that all course organisers apply prospectively for accreditation of the event and for credit point assessment. For repeated courses, renewed accreditation and CPD approval should be required.

- NMOs should notify course organisers as to the number of Category 1 credit points awarded, and this figure should be included in the advertisement of the event.

- Course organisers should provide the participants with documentation describing course content and, if combined with examination, their results.

- Each participant in a Category 1 activity should retain documentation which includes the content of the course and the results of an examination, if applicable.

- Category 1 events should be classified into events with and without examinations. Basically, one full hour of educational activity should correspond to $1 \mathrm{cp}$. However, events with examinations should be ranked higher (e.g. 1.5-2 times higher).

- A total of 100-150 cp of Category 1 is recommended per 5-year cycle. (This corresponds roughly to attending a 2-day meeting twice a year.) If an NMO feels that it needs to differ from this recommendation, at least $50 \mathrm{cp}$ per 5-year cycle (corresponding roughly to attending one 2-day meeting per year) should be expected.

\section{Category 2 credit points}

Due to the large variety and differing local availability of Category 2 events, EFOMP is recommending that the following classes of CPD activities may be considered. To allow for greater flexibility in setting up a National CPD scheme, the credit points may vary within a range of $\pm 30 \%$ of the recommended value.

- Formal local hospital educational activities, e.g. attendance at lectures, seminars, and regular organised teaching activities: $1 \mathrm{cp}$ per full lecture-hour or per meeting. Up to $5 \mathrm{cp}$ per year.

- On the job training activities and experiences, e.g. development of interpersonal skills, time management, leadership: Up to $5 \mathrm{cp}$ per year.
- Planned self-directed learning, e.g. reading of textbooks, journals, including computer-based "distance learning facilities": up to $5 \mathrm{cp}$ per year.

- Preparation and delivery of formal lecture or seminar: up to 2 $\mathrm{cp}$ for the first time presentation and $1 \mathrm{cp}$ for a repeated presentation. Up to $20 \mathrm{cp}$ per cycle.

- Visits to other departments for special training: $1 \mathrm{cp}$ per day up to $5 \mathrm{cp}$ per year.

- Publication of (a) a paper in a recognised scientific journal: up to $20 \mathrm{cp}$ per paper, depending on the type of the journal (e.g. peer reviewed/non-peer reviewed) and on the contribution of the author (main author, co-author); (b) Participation in writing a textbook: up to $50 \mathrm{cp}$ per complete book, depending on the size of the book, and up to $20 \mathrm{cp}$ per book chapter contribution and number of authors. Up to $50 \mathrm{cp}$ per cycle.

- Oral or poster presentation at a congress: up to $10 \mathrm{cp}$ per oral presentation, and up to $5 \mathrm{cp}$ per poster presentation, depending on the type of congress (international, national, regional) and the authorship (main author or co-author). Up to $50 \mathrm{cp}$ per cycle.

- Implementation of new technologies/procedures with a significant impact on medical physics: up to $5 \mathrm{cp}$ per activity and 10 cp per year for a documented implementation and development of new technologies and procedures, depending on the complexity of the technology.

- Active membership in task groups relevant to medical physics (working groups, standardisation committees, editorial boards of international scientific journals and equivalent): up to $5 \mathrm{cp}$ per membership and year, depending on type of group (international, national, regional, local) and scientific relevance (dosimetry protocols, equipment standardisation, radiation protection, etc.), altogether not more than $10 \mathrm{cp}$ per year.

- Expert assignments on international level up to $10 \mathrm{cp}$ per activity and up to $20 \mathrm{cp}$ per cycle.

- Organisation of scientific events (meetings, congresses, conferences): up to $20 \mathrm{cp}$ per event if international and up to $10 \mathrm{cp}$ for national. Up to $20 \mathrm{cp}$ per cycle.

Appendix B gives an example of a suitable credit point system.

\section{Summary}

The guidelines presented here constitute a set of general requirements on the design of the CPD scheme itself and the credit point system. CPD should be assessed on the basis of a 5-year cycle, and a total of 200-300 credit points should be earned during this period. Two categories of CPD activities are introduced, attendance at pre-assessed courses, conference or congresses (Category 1 ) and self-directed learning (Category 2), and the credit points should be about equally balanced between both categories. Category 1 credit points should be awarded on the general basis of one credit point per full course hour. Category 2 credit points should be awarded according to the relative importance and relevance of $\mathrm{CPD}$, for example: formal local hospital education activities, reading of textbooks and articles, lecturing, training visits, publications, congress contributions, implementation of new technologies and active membership in task groups.

EFOMP recommends that NMOs and European Societies involved in medical physics activities encourage participation in CPD schemes, to the benefit of the individual medical physicist, the employer, the patient and the medical physics profession. All beneficiaries should share the responsibility for CPD resources, i.e. the individual medical physicists, their employers, professional bodies and public education and training bodies. 


\section{Acknowledgements}

The Authors acknowledge the help of the EFOMP Officers in compiling this Policy Statement.

\section{Appendix A The necessity of CPD in medical physics}

CPD is the planned acquisition of knowledge, experience and skills (both technical and personal) required for professional practice throughout one's working life. CPD is therefore at the heart of the professional ideal. CPD is also necessitated by the pace of change of medical technology and methods.

The medical physicist is responsible in his/her area of expertise for equipment, techniques and methods used in routine as well as new clinical services. His/her personal store of knowledge, skills and competence must be updated to match the pace of change in medical technology, techniques and procedures.

At the very minimum CPD can be seen as necessary to the individual, the employer and the profession in order to maintain competence and to protect against challenges from competitors or even the courts. On a more positive note, the individual gains stimulation, job satisfaction and prospects for promotion, the profession gains repute and status, whilst the employer enjoys good staff morale and a positive attitude towards the introduction of new services. The patient, and thereby the public in general, benefits by good scientific and technical support for medical procedures, and the introduction of new techniques to routine practice.

There is no stage in the medical physicist's career where learning is complete. As one's career progresses, responsibilities grow and the knowledge base adapts to encompass new techniques, so that the need for CPD increases rather than decreases. Thus all medical physicists who have completed their basic education and training should be involved in CPD.

As already mentioned in Introduction individual medical physicist's CPD should be in line with his/her professional activities and registration level and his/her ambitions to achieve a higher professional level [6].

What constitutes continuing professional development?

CPD encompasses all activities which extend knowledge, skills, competence and the personal qualities required to deliver services. Common activities include:

- Attending and/or contributing to training courses, seminars, workshops, etc., as well as scientific meetings such as conferences, professional sessions.

- Self-study, research and publication (regular reading of journals, textbooks, standards literature, legislation, etc., and contributing to such literature).

- Service innovation (adapting or creating protocols, introduction of new services, etc.) education and training of medical physicists and related professions.

Each individual medical physicist should plan in advance for each calendar year, their professional development plan for the coming year, using as a guide the personal CPD cycle presented in Fig. A1. The final professional development record should be submitted to the national CPD scheme for evaluation and recording in the individual medical physicist's CPD record.

\section{Resources for continuing professional development}

CPD requires resources in the form of finance, time, education and training expertise. All beneficiaries (the individual, the employer, the public and the professional body) must share the responsibility for providing the necessary resources. The individual, in tandem with the employer, contributes time and finance towards his/her individual CPD programme, whilst the professional body organises workshops, conferences, etc., as well as developing

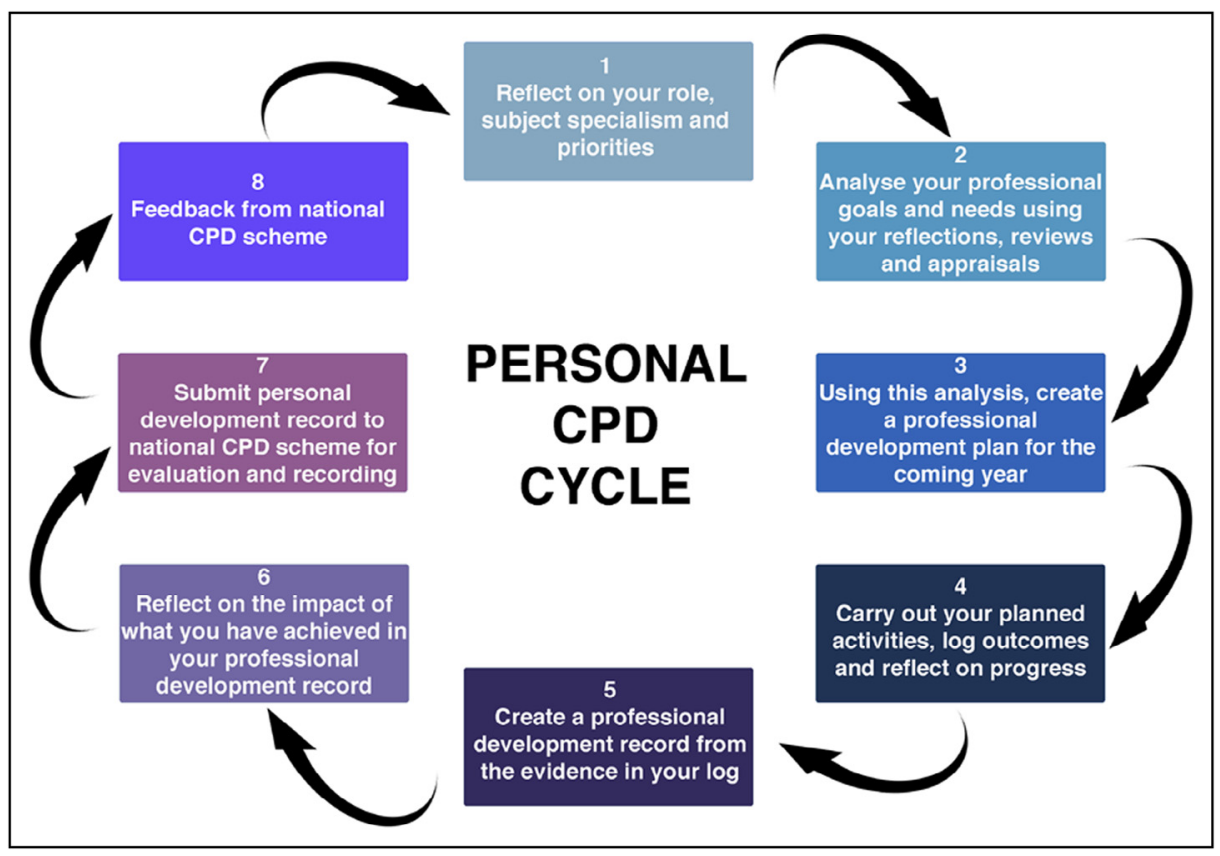

Figure A1. The eight steps of the personal CPD cycle. 
and administering a formal CPD scheme. The public contribution emanates mainly from the expertise and resources of public teaching and training institutes. The latter satisfies not only the public's duty to CPD, but also the educational institute's duty towards support and maintenance of its "products", i.e. the graduate workforce.

\section{Recommendations}

All medical physicists should be involved in CPD after qualification.

Formal CPD schemes should be developed to recognise individual effort.

Formal CPD schemes should set out clear objective guidance for the extent of CPD to be achieved within a defined timescale and as necessary at defined European Qualification Framework (EQF) levels [6].

Renewal of professional registration should be linked to CPD performance.

The resources for CPD should be provided by the individual, the professional body, the employer and public education and training bodies.

\section{Conclusions}

CPD is vital to the ideal of professionalism. It promotes excellence within the profession and protects the profession and the public against incompetence. Within the medical physics profession CPD is vital to embrace the pace of change occurring in medical practice.

Formal, standardised CPD schemes foster the status and integrity of the medical physics profession. CPD also benefits the individual medical physicist by maintaining competence and competitiveness, by promoting job satisfaction and career prospects. It benefits employers by ensuring minimal standards, and by encouraging competent, highly motivated staff, who can readily adapt to new techniques and practices. It benefits patients and therefore the public as a whole, by encouraging a high standard of technical and scientific support for patient care.

CPD requires commitment, time, finance and training expertise. The responsibility for CPD resources must be shared by all beneficiaries, i.e. individual medical physicists, their employers, professional bodies and public training and education institutions. Failure to implement CPD results in poor standards of patient care. Thus the costs of not involved in CPD far outweigh the costs of CPD.

\section{Appendix B An example of a suitable credit point system}

Please note that the example below does not cover all the categories or sub-categories of activities that should be covered by a modern CPD scheme for Medical Physicists and also the credit points allocated to each activity may not be appropriate for all NMOs. The example below should not be copy pasted into any national CPD scheme.

\begin{tabular}{|c|c|}
\hline \multicolumn{2}{|l|}{ An example of a suitable credit point system based on 2 categories of activities } \\
\hline Category 1 activities & \\
\hline $\begin{array}{l}\text { Attendance at pre-assessed courses (i.e. lectures, scientific meetings, workshops, } \\
\text { refresher/training courses), national and international }\end{array}$ & $\begin{array}{l}\text { General rule, } 1 \mathrm{cp} / \mathrm{h} \\
\text { events with examination } 2 \mathrm{cp} / \mathrm{h}\end{array}$ \\
\hline Total number of Cat. 1 credit points & $100-150$ cp per 5 -year cycle \\
\hline Category 2 activities & \\
\hline $\begin{array}{l}\text { Attendance at formal local hospital educational activities (e.g. lectures, seminars, } \\
\text { regular organised teaching activities) }\end{array}$ & $1 \mathrm{cp} /$ meeting or $1 \mathrm{cp} /$ lecture-hour. Maximum $10 \mathrm{cp} /$ year \\
\hline $\begin{array}{l}\text { On the job training activities and experiences, e.g. includes development of } \\
\text { interpersonal skills, time management }\end{array}$ & Up to $10 \mathrm{cp} /$ year \\
\hline $\begin{array}{l}\text { Planned self-directed learning (e.g. reading of textbooks, journals, } \\
\text { including “distance learning facilities") }\end{array}$ & Up to $10 \mathrm{cp} /$ year \\
\hline Preparation and delivery of formal lecture or seminar & $\begin{array}{l}10 \mathrm{cp} \text { for first time presentation, } 2 \mathrm{cp} \text { for repeated presentation. } \\
\text { Maximum } 15 \mathrm{cp} / \text { year }\end{array}$ \\
\hline Special training visits to other departments & Up to $5 \mathrm{cp} /$ year \\
\hline Publication of & (a) 2-20 cp, depending on the type of journal (e.g. peer-reviewed or not) and \\
\hline $\begin{array}{l}\text { (a) a paper in a recognised scientific journal } \\
\text { (b) a textbook }\end{array}$ & $\begin{array}{l}\text { on the contribution of the author } \\
\text { (b) 5-30 cp, depending on the authorship and the size of the contribution } \\
\text { Maximum } 30 \mathrm{cp} / \text { year }\end{array}$ \\
\hline Oral or poster presentation at congress & $\begin{array}{l}2-10 \mathrm{cp} \text { per presentation, depending on type of congress (international, } \\
\text { national, regional) and authorship (single author, co- author) } \\
\text { Maximum } 15 \mathrm{cp} / \text { year }\end{array}$ \\
\hline Implementation of new technologies/procedures & $\begin{array}{l}\text { Up to } 5 \mathrm{cp} \text { per activity and } 10 \mathrm{cp} \text { per year for a documented implementation } \\
\text { and development of new technologies and procedures, depending on the } \\
\text { complexity of the technology }\end{array}$ \\
\hline $\begin{array}{l}\text { Active membership in task groups (working groups, standardisation } \\
\text { committees and equivalent) }\end{array}$ & $\begin{array}{l}\text { Up to } 5 \mathrm{cp} \text { per membership and year, depending on type of group (international, } \\
\text { national, regional, local) and scientific relevance (dosimetry protocols, equipment } \\
\text { standardisation, radiation protection, etc.) } \\
\text { Maximum } 15 \mathrm{cp} / \text { year }\end{array}$ \\
\hline Total number of Cat. 2 credit points & $100-150$ cp per 5 -year cycle \\
\hline Total number of credit points & 250 cp per 5 -year cycle \\
\hline
\end{tabular}

\section{References}

[1] Caruana CJ, Christofides S, Hartmann GH. EFOMP policy statement no. 12.1, recommendations on medical physics education and training in Europe 2014. Phys Med 2014;30:598-603.

[2] Christofides S, Isidoro J, Pesznyak C, Bumbure L, Cremers F, Schmidt WFO. EFOMP Policy Statement No. 6.1 "Recommended Guidelines on National Registration Schemes for Medical Physicists", 2016.

[3] Council Directive 2013/59/EURATOM of 5 December 2013 laying down basic safety standards for protection against the dangers arising from exposure to ionising radiation, and repealing Directives 89/618/Euratom, 90/641/Euratom, 6/29/Euratom, 97/43/Euratom and 2003/122/Euratom, OJ L13, 2014, pp. 173.

[4] IAEA Safety Standards, General Safety Requirements Part 3 No. GSR Part 3 "Radiation Protection and Safety of Radiation Sources: International Basic Safety Standards", STI/PUB/1578, IAEA, Vienna, 2014.

[5] EC RP 174, "Guidelines on Medical Physics Expert". Freely downloadable from http://ec.europa.eu/energy/sites/ener/files/documents/174.pdf; 2014.

[6] Parliament and Council Recommendation 2008/C 111/01. Official Journal of the European Union, C111; 2008. 